\title{
Michał HRABIA
}

Uniwersytet Śląski

michal_hrabia@tlen.pl

\section{LE VERBE $A L L E R$ \\ DANS LA TRADUCTION AUTOMATIQUE. QUELQUES REMARQUES SUR L'ITÉRATIVITÉ ÉTUDIÉE DANS LE CADRE DE L'APPROCHE ORIENTÉE OBJETS}

\section{À TITRE D'INTRODUCTION}

L'épanouissement des nouvelles technologies, observé depuis quelques dizaines d'années, a permis de sortir de 'l'ombre scientifique' à toute une série de disciplines suspendues jusqu'alors dans l'imaginaire. Tel est le cas de la traduction automatique qui, après une longue période de désespoir et de stagnation, connaît aujourd'hui son essor prodigieux.

L'impossible est devenu possible, et voire ordinaire, pourrait-on dire. Néanmoins, quoique la puissance de calcul et la mémoire des ordinateurs ne constituent plus aucun obstacle pour le traitement automatique des langues, il est indéniable que les résultats de la traduction effectuée par les machines sont toujours loin d'être parfaits. Cela se traduit, paraît-il, par le fait que le progrès dans le domaine de l'informatique n'a pas été accompagné d'un progrès suffisant dans le domaine de la linguistique computationnelle. En effet, il manque toujours de descriptions formelles complètes et exhaustives des langues naturelles qui pourraient être informatiquement implémentables, c'est-à-dire compréhensibles pour la machine. La langue naturelle avec toutes ses subtilités et ses nuances (parmi lesquelles on compte p. ex. le fameux phénomène de la polysémie) continue à s'échapper à une formalisation rigoureuse, mais nécessaire $\mathrm{du}$ point de vue du traitement informatique, en constituant ainsi une véritable barrière, parfois impossible à surmonter, pour les traducteurs automatiques (cf. p. ex. Żłobińska-Nowak 2013). 
La présente communication cherche à contribuer à la destruction, ne serait-ce que partielle, de ce barrage. Dans ce qui suit, nous allons nous concentrer sur l'un des problèmes qui empêche la traduction automatique efficace $d u$ français vers le polonais : la question de l'itérativité des verbes de mouvement. En nous appuyant sur l'exemple du mot aller, nous allons montrer comment on peut examiner et décrire ce phénomène linguistique de manière qu'il soit traduisible automatiquement.

\section{ITÉRATIVITÉ DANS LE SYSTÈME VERBAL POLONAIS}

On sait que la plupart des infinitifs polonais apparaissent sous deux formes morphologiquement distinctes appelées dans la tradition grammaticale 'perfective' et 'imperfective' (p. ex. czytać - forme imperfective $\mathrm{du}$ verbe lire, przeczytać - forme perfective). Toutefois, la différenciation aspectuelle du lexique verbal polonais ne s'arrête pas ici. En polonais, tout comme dans les autres langues slaves (sauf le bulgare et le macédonien), il existe un ensemble clos de verbes dits 'de mouvement' qui, à l'intérieur de l'imperfectif, distinguent supplémentairement deux 'sous-aspects', dont le premier exprime le mouvement unidirectionnel (p. ex. jechać, iść, plynąć) et le deuxième - le mouvement multidirectionnel (p. ex. jeździć, chodzić, pływać). Le tableau ci-dessous (tab. 1) présente la liste communément admise de ces verbes (cf. p. ex. Kuszmider 1999 : 42, Włodarczyk $1980: 49$ ).

La détermination de la direction du mouvement n'est pas pourtant un critère fiable ou suffisant pour décrire les verbes de mouvement dans tous leurs différents emplois. En effet, en définissant l'opposition du type jechać vs jeździć, il est indispensable de prendre en considération un autre critère, à savoir : l'itérativité. Par conséquent, les verbes du type jeździć, chodzić sont le plus souvent considérés comme multidirectionnels et/ou itératifs, et les verbes jechać, iść comme unidirectionnels et/ou non itératifs (sémelfactifs).

La notion de l'itérativité demande un peu plus d'explication. Quoique les linguistes ne soient pas unanimes quant à l'interprétation précise de ce phénomène linguistique, dans toutes les définitions proposées on peut facilement retrouver un 'noyau commun', un certain invariant qui s'exprime par l'idée d'une répétition (réelle ou potentielle) des actions semblables (cf. p. ex. Antinucci, Gebert 1977 : 38, Karolak 2001 : 537-539, Stawnicka $2007: 25)$. 
TABLEAU 1. La liste des verbes de mouvement en polonais

\begin{tabular}{|l|l|l|}
\hline $\begin{array}{c}\text { Verbe } \\
\text { unidirectionnel }\end{array}$ & $\begin{array}{c}\text { Verbe } \\
\text { multidirectionnel }\end{array}$ & \multicolumn{1}{c|}{ Équivalent français } \\
\hline jechać & jeździć & aller (en véhicule) \\
\hline iść & chodzić & marcher, aller (à pied) \\
\hline biec/biegnąć & biegać & courir \\
\hline lecieć & latać & voler \\
\hline nieść & nosić & porter \\
\hline płynąć & pływać & nager \\
\hline leźć & łazić & grimper, aller (fam.) \\
\hline wieźć & wozić & transporter, conduire (en voiture) \\
\hline pełznąć & pełzać & ramper \\
\hline gnać/gonić & ganiać & poursuivre \\
\hline wlec (się) & włóczyć (się) & (se) traîner \\
\hline
\end{tabular}

Pour les besoins de cet article, nous adoptons la définition formulée par Jean-Pierre Desclés et Zlatka Guentchéva. Afin de décrire l'itérativité et de rendre compte de ses propriétés aspectuelles, ils proposent d'introduire le concept de 'classe d'événements' qui représente une série d'occurrences identiques du même événement (Desclés, Guentchéva 1997 : 161). Ces occurrences se déploient sur des intervalles fermés et sont séparées les unes des autres par des situations statiques (états de 'non-déplacement') (fig. 1).

FIG. 1. La représentation topologique de la classe d'occurrences d'événements itérés

$$
[[--] \ldots \ldots .[--] \ldots \ldots . .[--] \ldots \ldots \ldots \ldots \ldots \ldots \ldots . . .[--][
$$

Il faut encore réfléchir sur le statut de l'itérativité dans la description du fonctionnement des verbes du type jeździć, chodzić en polonais. Il semble que la tradition linguistique polonaise accentue en particulier le caractère multidirectionnel des verbes en question (cf. p. ex. Piernikarski 1969 : 103-104, Laskowski 1998 : 165) en repoussant, pour ainsi dire, la notion d'itérativité en arrière-plan. Nous nous permettons à signaler que, selon nous, une telle interprétation est fort abusive. En fait, dans la plupart des cas, l'itérativité seule s'avère suffisante pour décrire les emplois 
des verbes du type jeździć, chodzić, lors même qu'ils relèvent (au moins au premier abord) de la multidirectionnalité de mouvement. On peut donc constater que l'itérativité est une notion primaire qui définit les verbes jeździć, chodzić de façon universelle, tandis que la multidirectionnalité n'est qu'un trait secondaire qui se manifeste supplémentairement dans un nombre limité d'emplois. C'est pourquoi nous préférons utiliser les dénominations 'verbes itératifs vs sémelfactifs' au lieu des 'verbes unidirectionnels vs multidirectionnels'.

\section{ANALYSE ORIENTÉE OBJETS}

Il est évident que l'itérativité, en tant que catégorie dont on ne connaît pas d'équivalents dans des langues autres que slaves, pose bien des difficultés dans la pratique de la traduction automatique. Il est temps que nous envisagions ce phénomène du point de vue du traitement automatique afin d'essayer de remédier aux problèmes qu'il pose dans ce domaine.

Le cadre théorique dans lequel s'inscrit notre étude - l'approche orientée objets (l'AOO) - a été élaboré à l'Université de Silésie par Wiesław Banyś (2002ab). Inspirée en autre de la théorie des classes d'objets de Gaston Gross (1994, 2008), cette conception place au centre de la description linguistique la notion de la 'classe d'objets', celle-ci étant définie comme un ensemble d'objets (c'est-à-dire un ensemble d'unités linguistiques) qui partagent un certain nombre d'opérations et d'attributs (Banyś 2002a : 22). L'approche orientée objets trouve son application pratique dans la désambiguïsation des sens des mots, une méthode simple et efficace qui permet de créer des schémas formels (appelés 'schémas syntaxico-sémantiques') décrivant d'une façon claire et univoque des emplois particuliers d'un mot donné (cf. p. ex. Banyś 2005, Hrabia 2011). C'est justement cette méthode que nous allons employer dans la suite de la présente communication afin de découvrir et de schématiser les conditions linguistiques (syntaxiques et sémantiques) déterminant le choix de la forme sémelfactive jechać ou itérative jeździć dans la traduction du verbe aller $\mathrm{du}$ français vers le polonais. Étant donné que nous ne voulons pas que la description de l'opposition itératif vs sémelfactif soit influencée par des facteurs modo-temporels (qui pourraient perturber et compliquer inutilement la désambiguïsation), nous allons limiter notre corpus uniquement aux formes du présent grammatical dont les valeurs tempo- 
relles sont grosso modo les mêmes en français et en polonais (cf. Gniadek 1979 : 97).

\section{EMPLOI I}

(1) Mon épouse et moi nous allons régulièrement en Sardaigne dans la région d'Oristano.

(2) D'habitude, je vais en Californie pendant les vacances d'été.

(3) Je vais toujours en voiture à mon travail parce que le bâtiment est très loin de chez moi. Il y a un grand parking près de mon travail.

(4) Mon autre passion, c'est tout ce qui touche à la culture. Critique théâtre pour un site spécialisé depuis quelques années, je vais tous les ans à Avignon et je m'apprête à lancer un site spécialisé sur la danse en collaboration avec Pierre Assouline.

jeździmy (jeździć)
$j e \dot{z} d \dot{z} e$ (jeździć)
$j e \dot{z} d \dot{z} e$ (jeździć)
jeżḋę (jeździć)

On voit clairement que les adverbes soulignés font que l'action prédiquée par le verbe aller acquiert un caractère répétitif. Du point de vue cognitif, elle se compose de nombreux actes de mouvement qui sont interrompus par des phases statiques de non-déplacement. Le choix de l'équivalent jeździć en polonais est donc indiscutable. L'emploi analysé peut être formalisé à l'aide du schéma suivant (cf. Hrabia 2014 : 50-53) :

\section{JEŹDZIĆ (SCHÉMA I)}

fr.

$\langle$ marqueur de fréquence $\rangle-\mathbf{X}-[$ ANM hum $]-\operatorname{aller}_{\text {[prés.] }}-(e n / \grave{a}-\mathbf{Y}-$ [CONC $\langle$ moyen de transport terrestre $\rangle])-\{($ de $-\mathbf{Z}-[$ CONC $\langle$ lieu $\rangle])-$ $\grave{a} /$ en/jusqu'à/sur/dans/vers/en direction de - W - [CONC $\langle$ lieu $\rangle]\} \bigvee\{$ chez - W - [ANM hum]\}

$/ / \wedge\langle$ marqueur de fréquence $\rangle \in \operatorname{aller}(X) / /$

pol.

$\langle$ określnik częstotliwości $\rangle-\mathbf{X}-$ [ANM hum] - jeździć́ [teraź.] $-(\mathbf{Y}-[$ [CONC (środek transportu naziemnego $\rangle])-\{(z-\mathbf{Z}-[\mathrm{CONC}\langle$ miejsce $\rangle])-$ do $/ a \dot{z}$ do/w/na/nad/w stronę/w kierunku - W - [CONC $\langle$ miejsce $\rangle]\} \backslash\{$ do $-\mathbf{W}-$ [ANM hum]\}

$/ / \wedge\langle$ określnik częstotliwości $\rangle \in$ jeżdzić $(X) / /$ 
La position du sujet $X$ est remplie par la classe d'objets [ANM hum] ${ }^{1}$, la position du complément $\mathrm{Y}$ - facultativement par la classe [CONC 〈moyen de transport terrestre〉] et la position du complément $\mathrm{W}$ - (obligatoirement) par la classe [CONC $\langle$ lieu $\rangle]$ ou [ANM hum]. Remarquons que si la position $W$ est remplie par la classe [CONC $\langle$ lieu $\rangle]$, notre schéma prévoit aussi la position $\mathrm{Z}$ (facultativement remplie par la classe [CONC $\langle$ lieu $\rangle$ ]).

Dans le schéma apparaît aussi la classe $\langle$ marqueur de fréquence $\rangle$. Notons que cette classe ne doit pas obligatoirement précéder le sujet ; son emplacement au début du schéma n'est qu'une simplification formelle. Sous le nom de /marqueur de fréquence〉 on comprend les adverbes de fréquence tels que souvent, d'habitude ${ }^{2}$, mais aussi les constructions suivantes : chaque 〈unité de la mesure du temps〉 (p. ex. chaque jour), tous (toutes) les 〈unité de la mesure du temps〉 (p. ex. tous les ans), nombre fois par 〈unité de la mesure du temps〉 (p. ex. deux fois par semaine) et un jour par 〈unité de la mesure du temps $\rangle$ (p. ex. un jour par semaine).

La formule $\langle$ marqueur de fréquence $\rangle \in$ aller $(X)$, quant à elle, vise à accentuer que le schéma est plus adéquat lorsque le marqueur de fréquence s'applique directement à la proposition créée par le prédicat aller.

\section{EMPLOI II}

(1) Comme toujours, nous allons à Mooréa passer quelques jours.

(2) Pour Noël je vais comme d'habitude à Paris, donc sûrement plein de photos de la plus belle ville d'Europe!

(3) Nous allons comme d'habitude à Bigulia (à côté de Bastia) dans notre "petit», mais non moins confortable pied à terre au bord de la mer.

(4) - Moi, comme toujours, je vais à Focsani voir ma mère, elle est toute seule et elle m'attend. À peine prononça-t-elle ces mots qu'elle les regretta. [M. Belis]

jedziemy (jechać)
jadę (jechać)
jedziemy (jechać)
jadę (jechać)

1 Explication des abréviation : ANM - animé, hum - humain, CONC - concret, ABSTR - abstrait.

2 Dans notre analyse les adverbes et les locutions adverbiales possèdent, tout comme les noms, le statut d'objets. Cela reste en accord avec les principes de l'approche orientée objets selon lesquelles « l'objet » est une notion fonctionnelle, et en tant que telle, peut être représenté par les mots ou les constructions appartenant à des catégories grammaticales différentes. 
Tous les exemples ci-dessus contiennent des expressions du type comme $+\langle$ marqueur de fréquence $\rangle$ (p. ex. comme toujours, comme d'habitude). On pourrait croire que la présence de marqueurs proprement itératifs provoquera tout naturellement la traduction du verbe aller par la forme jeździć. Et pourtant, le seul équivalent correct pour toutes les occurrences présentées est la forme sémelfactive jechać. En fait, la ressemblance entre les adverbes toujours, d'habitude d'un côté, et comme toujours, comme d'habitude de l'autre n'est qu'une similarité apparente. Il s'avère que ces deux groupes d'adverbes influencent la proposition aller $(X)$ d'une façon complètement différente : tandis que les adverbes toujours, d'habitude mettent l'accent sur le caractère habituel, répétitif du mouvement, les adverbes comme toujours, comme d'habitude focalisent l'attention sur une réalisation concrète et unique de l'action a priori habituelle. En effet, en disant Je vais comme d'habitude à Paris, le locuteur veut accentuer qu'il s'agit d'un déplacement concret, unique, mais qui s'est déjà plusieurs fois réalisé et qui a le potentiel de se réaliser à l'avenir.

Le schéma décrivant cet emploi du verbe aller se présente comme suit :

\section{JECHAĆ (SCHÉMA II)}

fr.

comme $\langle$ marqueur de fréquence $\rangle-\mathbf{X}-\left[\right.$ ANM hum] $-\operatorname{aller}_{[\text {prés.] }}-(e n / \grave{a}-\mathbf{Y}$

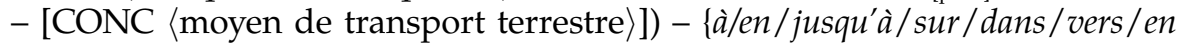
direction de - W - [CONC $\langle$ lieu $\rangle]\} \bigvee\{$ chez - W - [ANM hum $]\}$

pol.

jak 〈określnik częstotliwości $\rangle-\mathbf{X}-$ [ANM hum] - jechać́ [teraź.] $-(\mathbf{Y}-$ [CONC 〈środek transportu naziemnego $\rangle])-\{$ do/aż do/w/na/nad/w stronę/w kierunku - W - [CONC $\langle$ miejsce $\rangle]\} \bigvee\{$ do $-\mathbf{W}-[$ ANM hum $]\}$

\section{EMPLOI III}

(1) Je découvre aussi l'histoire locale, les écrits de René Botto sur l'Isle-Adam, je vais aux conférences de la société historique de Pontoise...

(2) Nous allons aux réunions et nous appelons les gens qui participent au programme pour discuter de questions de rétablissement.

(3) Je vais à la conférence internationale du développement durable appelée Rio+20 pour manifester mon

$j e \dot{z} d \dot{z} e$ (jeździć)

jeździmy (jeździć)

jadę (jechać) 
désaccord avec le projet concocté par les puissants de la planète.

(4) Je vais au concert de Depeche Mode à Nancy. Je propose un covoiturage au départ de Strasbourg, j'ai 3 places. Si quelqu'un est intéressé, envoyez-moi un mail.

Remarquons que, quoique toutes les phrases ci-dessus réalisent des schémas syntaxico-sémantiques apparemment identiques, leur caractère (du point de vue de leur traduction en polonais) varie - il est itératif dans les exemples (1) et (2) et sémelfactif dans (3) et (4). Il s'avère que cela est dû au nombre grammatical de l'instance de la classe d'objets occupant la position du complément : si elle est mise au pluriel, l'action acquiert un caractère itératif, sinon, l'action est considérée comme sémelfactive. Une telle constatation est conforme à notre intuition linguistique : il est évident que lorsque l'on va aux concerts (et non pas à un concert concret) nos voyages sont plus ou moins réguliers, répétitifs sur l'intervalle topologique, donc itératifs.

Les schémas décrivant l'emploi analysé se présentent alors de la façon suivante :

\section{JECHAĆ (SCHÉMA III ${ }_{A}$ )}

fr.

$\mathbf{X}-\left[\right.$ ANM hum] - aller $_{\text {[prés. }}-(e n / \grave{a}-\mathbf{Y}-[$ CONC $\langle$ moyen de transport terrestre $\rangle])-\grave{a} / e n-\mathbf{W}-[$ ABSTR (événement culturel/sportif $\rangle]_{\text {[sing.] }}$

pol.

$\mathbf{X}-\left[\right.$ ANM hum] - jechać́ ${ }_{\text {[teraź.] }}-(\mathbf{Y}-$ [CONC 〈środek transportu naziemnego $\rangle])-n a-\mathbf{W}-[\text { ABSTR }\langle\text { wydarzenie kulturalne/sportowe }\rangle]_{[\text {poj.l }}$

JEŹDZIĆ (SCHÉMA III ${ }_{B}$ )

fr.

$\mathbf{X}-\left[\right.$ ANM hum] $-\mathbf{a l l e r}_{[\text {prés. }}-(e n / \grave{a}-\mathbf{Y}-[$ CONC $\langle$ moyen de transport terrestre $\rangle])-\grave{a} / e n-\mathbf{W}-[\text { ABSTR }\langle\text { événement culturel/sportif }\rangle]_{[p l .]}$

pol.

$\mathbf{X}-[$ ANM hum $]$ - jeździć́ ${ }_{\text {[teraź. }]}-(\mathbf{Y}-$ [CONC 〈́́rodek transportu naziemnego $\rangle])-n a-\mathbf{W}-[\text { ABSTR }\langle\text { wydarzenie kulturalne/sportowe }\rangle]_{[\mathrm{mn} .]}$

Néanmoins, il est possible d'imaginer la situation où le sujet parlant effectue un seul mouvement malgré la pluralité des événements auxquels il assiste. Voici quelques exemples : 
(5) Heures de ménage ce matin et 3 lessives; un allerretour au club hippique pour le premier cours de la saison de l'Enfant-Chérie (un tea-time papotage pendant le cours...) ; la préparation d'un repas pour les deux enfants puisque l'Homme a des matches de hand ce soir (non, je n'ai pas fait de faute, il va à DEUX matches).

(6) Au retour nous allons à deux conférences de presse. Le ministre de l'information fait ses annonces de réussites au combat en qualifiant les troupes anglaises et américaines de mercenaires.

jedzie (jechać)

jedziemy (jechać)

Il est incontestable que dans les extraits ci-dessus le verbe aller doit être obligatoirement traduit en polonais à l'aide de la forme sémelfactive jechać, bien que la classe d'objets [ABSTR 〈événement culturel/sportif〉] soit mise au pluriel. Il paraît donc que la langue polonaise rejette la forme itérative lorsque devant la classe d'objets [ABSTR 〈événement culturel/sportif $\rangle]$ apparaît un chiffre concret (comparez p. ex. Jeżdże na mecze vs. ? Jeżdżę na dwa meczẻ). Voici le schéma :

\section{JECHAĆ (SCHÉMA IIIC)}

fr.

$\mathbf{X}-\left[\right.$ ANM hum] - aller $_{\text {[prés. }]}-(e n / \grave{a}-\mathbf{Y}-$ [CONC $\langle$ moyen de transport terrestre $\rangle]$ ) $-\grave{a} / e n-\mathbf{W}-\mid$ chiffre| [ABSTR $\langle$ événement culturel/sportif $\rangle]_{[p 1 .]}$ pol.

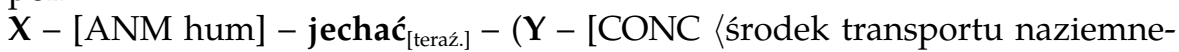
go $\rangle)]-n a-\mathbf{W}-\mid$ cyfra| [ABSTR $\langle$ wydarzenie kulturalne/sportowe $\rangle]_{[m n .]}$

\section{EMPLOI IV}

(1) Je vais de ville en ville à la recherche d'enfants qui ont besoin d'aide.

(2) Ils vont de pays en pays, souvent mal orientés ; parfois on leur donne des besognes secondaires, mais certains d'entre eux parviennent à percer et finissent même par s'imposer grâce à leur intelligence, leur ténacité et leur évidente bonne volonté.

jeżḋę (jeździć)

jeżḋ̇a (jeździć)

3 Le signe ? marque une construction plutôt inacceptable. 
(3) Ils vont de ville en ville, récoltent les gants hors d'usage et oubliés afin de raconter des histoires dans leur grande boîte à gants.

(4) Il va de ville en ville, et note dans son vieux carnet toutes les recettes qu'il découvre.

$j e \dot{z} d \dot{z} q(j e z ́ d z i c ́)$

jeździ (jeździć)

Dans les exemples présentés, le verbe aller choisit comme son équivalent polonais la forme itérative jeździć. Cela s'explique par la présence de l'expression de $\langle$ lieu $\rangle$ en $\langle l i e u\rangle$ qui, aussi bien en français qu'en polonais, introduit une idée de répétition des microactions de déplacement. En effet, le sujet ne se déplace pas uniquement d'un point $A$ vers $B$; après avoir atteint $B$, il continue son voyage en se déplaçant de $B$ vers $C$, puis de $C$ vers $D$, et ainsi de suite. Par conséquent, pour que l'on puisse comprendre ce que aller de $\langle$ lieu $\rangle$ en $\langle l i e u\rangle$ veut dire, on doit prendre en considération toutes les actions de déplacement effectuées. Cela 'visualise' bien le déploiement événementiel du prédicat aller dans ce contexte-là (cf. Borillo $2006: 4$ ) et justifie le choix de la forme indéterminée en polonais. Le schéma est le suivant :

JEŹDZIĆ (SCHÉMA IV)

fr.

$\mathbf{X}-[$ ANM hum $]-$ aller $_{\text {[prés.] }}-(e n / \grave{a}-\mathbf{Y}-[$ CONC $\langle$ moyen de transport terrestre $\rangle])-d e-\mathbf{Z}-[$ CONC $\langle$ lieu $\rangle]-e n-\mathbf{W}-[$ CONC $\langle$ lieu $\rangle]$

$/ / \mathrm{Z}_{\text {[LEX.] }}=\mathrm{W}_{\text {[LEX.] }} / /$

pol.

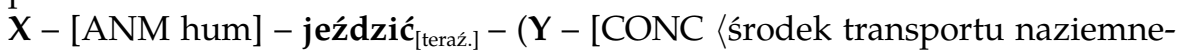
go $\rangle])-z / o d-\mathbf{Z}-[$ CONC $\langle$ miejsce $\rangle]-d o-\mathbf{W}-[$ CONC $\langle$ miejsce $\rangle]$

$/ / \mathrm{Z}_{\text {[LEX.] }}=\mathrm{W}_{\text {[LEX.] }} / /$

Il ne faut pas oublier que le schéma ci-dessus n'est adéquat que quand les positions $\mathrm{Z}$ et $\mathrm{W}$ sont remplies par les unités ayant la même forme morphologique $\left(Z_{[\text {[EX.] }}=W_{\text {[LEX.] }}\right)$.

\section{EN GUISE DE CONCLUSION}

Pour conclure, on peut dire que l'interprétation itérative du mot aller, telle qu'elle émerge de notre analyse, est conditionnée grosso modo par deux sortes de facteurs : 
- marqueurs temporels indiquant l'habitualité (facteur temporel) : schéma I,

- pluralité de destinations (facteur spatial) : schéma $\mathrm{III}_{\mathrm{B}}$, schéma IV.

Néanmoins, comme nous avons pu observer dans les schémas II et III $_{C}$, même de très petites modifications dans le tissu textuel (comme p. ex. introduction d'un chiffre) peuvent détourner complètement l'interprétation et provoquer le choix de l'équivalent sémelfactif en polonais. Afin de garantir la traduction correcte, il faut donc veiller à ce que les schémas syntaxico-sémantiques formulés au cours de la désambiguïsation soient aussi exhaustifs et complets que possible.

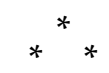

L'analyse présentée dans cet article peut être considérée comme la première description purement opérationnelle de l'itérativité créée exclusivement à des fins de traduction automatique de textes. Elle ne constitue qu'une partie du grand travail de la désambiguïsation orientée objets des verbes de mouvement effectué depuis quelques années dans le Département de Linguistique Appliquée et de Traduction à l'Université de Silésie.

\section{RÉFÉRENCES}

Antinucci F., Gebert L., 1977, «Semantyka aspektu czasownikowego», Studia gramatyczne I, 7-43.

Banyś W., 2002a, «Bases de données lexicales électroniques - une approche orientée objets. Partie I : Questions de modularité», Neophilologica 15, 7-28.

Banyś W., 2002b, «Bases de données lexicales électroniques - une approche orientée objets. Partie II : Questions de description", Neophilologica 15, 206-248.

Banyś W., 2005, « Désambiguïsation des sens des mots et représentation lexicale du monde », Neophilologica 17, 57-76.

Borillo A., 2006, «Partition, balayage, recouvrement total», Actes du Colloque La partition en langue et discours, [disponible en ligne le 31/07/2015] : http:// w3.erss.univ-tlse2.fr:8080/index.jsp?perso=borillo\&subURL=Borillo06Stras bourg.pdf.

Desclés J.-P., Guentchéva Z., 1997, «Aspects et modalités d'action (Représentations topologiques dans une perspective cognitive) », Studia Kognitywne 2, 145-173.

Gniadek S., 1979, Grammaire contrastive franco-polonaise, Warszawa. 
Gross G., 1994, "Classes d'objets et description des verbes », Langages 115, 15-30. Gross G., 2008, «Les classes d'objets », Lalies 28, 111-165.

Hrabia M., 2011, "Désambiguïsation des sens du prédicat adjectival farouche dans le cadre d'une approche orientée objets », Neophilologica 23, 150-167.

Hrabia M., 2014, «Wstęp do obiektowej analizy czasownika aller w konstrukcjach z określnikami temporalnymi dla celów tłumaczenia automatycznego francusko-polskiego », LingVaria 2 (18), 43-54.

Karolak S., 2001, «Iteratywność czy potencjalność? », in : Od semantyki do gramatyki. Wybór rozpraw, S. Karolak (éd.), Warszawa, 531-539.

Kuszmider B., 1999, Aspect, temporalité et modalité en polonais et en français. Étude contrastive, Paris.

Laskowski R., 1998, "Kategorie werbalne», in : Gramatyka współczesnego języka polskiego. Morfologia, R. Grzegorczykowa, R. Laskowski, H. Wróbel (éd.), Warszawa, 142-178.

Piernikarski C., 1969, Typy opozycji aspektowych czasownika polskiego na tle stowiańskim, Wrocław.

Stawnicka J., 2007, Aspekt - iteratywność - określniki kwantyfikacyjne, Katowice.

Włodarczyk H., 1980, "Czasowniki ruchu a kategoria aspektu w języku polskim », Polonica VI, 49-56.

Żłobińska-Nowak A., 2013, «Traduction automatique des langues - espérances et enjeux des outils appliqués », Neophilologica 25, 56-66.

\section{LE VERBE ALLER DANS LA TRADUCTION AUTOMATIQUE. QUELQUES REMARQUES SUR L'ITÉRATIVITÉ ÉTUDIÉE DANS LE CADRE DE L'APPROCHE ORIENTÉE OBJETS}

\section{Résumé}

Le but de cet article est de présenter les résultats de l'application de l'approche orientée objets de Wiesław Banyś dans la désambiguïsation de l'opposition intra-aspectuelle du type itérativité vs sémelfactivité. Après une courte introduction théorique à la question de l'itérativité des verbes de mouvement, l'auteur procède à l'étude des exemples concrets de quelques emplois du verbe aller et construit par la suite six schémas syntaxico-sémantiques déterminant la traduction polonaise du verbe analysé à l'aide de la forme itérative (jeździć) ou bien sémelfactive (jechać). Le format de description de l'itérativité employé dans cet article permet d'intégrer les résultats de la recherche dans les programmes de traduction automatique.

Mots-clés : traduction automatique, classe d'objets, verbes de mouvement, aspect verbal, itérativité 


\title{
VERB ALLER IN MACHINE TRANSLATION. SOME REMARKS ON THE ITERATIVITY STUDIED WITHIN THE OBJECT-ORIENTED APPROACH
}

\begin{abstract}
Summary
The aim of this paper is to present the results of the application of the object-oriented approach by Wiesław Banys in the disambiguation of the intra-aspectual opposition iterative vs semelfactive. After a brief theoretical introduction to the question of the motion verbs' iterativity, the author proceeds to study concrete examples of some uses of the French verb aller and creates six syntactic-semantic schemes conditioning the Polish translation of the analysed verb by the iterative (jeździć) or semelfactive form (jechać). The format of the description of the iterativity used in this paper allows to integrate the results of the research into the Machine Translation programs.
\end{abstract}

Key words: Machine Translation, object class, verbs of motion, verbal aspect, iterativity 